\title{
Performance of Eigensolvers in Modal Analyses of Nuclear Plant Building Structures
}

\author{
K.-R. Leimbach \\ Haverkampstr. 12, D.4630 Bochum-Linden, Germany \\ V.H. Engelke, N. Krutzik \\ Kraftwerk Union AG, Abteilung R621, Berliner Str. 295-299, D-6050 Offenbach, Germany
}

\section{SUMMARY}

The modal time history analysis of nuclear plant bullding structures subjected to dynamic excltations requires the computation of free vibration modes and frequencles. The models are extremely complex and are characterized by a large number of equations, a large half-bandwidth and a large number of modes and frequencies to be computed.

Several Improved elgensolution routines have been offered on the software market to address this situation. They are determinant search methods, subspace iteration methods with shifting, the Lanczos and the Householder QR-methods.

The large size of the models as well as the large number of modes and frequencles requires a thorough investigation of the performance characteristics of these elgensolution routines before a cholce 1 s made of the most sultable one. While their performance with carefully selected benchmark problems may be impressive their application to building models with unexpectedly clustered free vibration characteristics may pose a number of problems. These include lack of convergence, skipping of modes, exceeding demands on secondary storage capacities, exceeding allotted run times and progressive deterioration of accuracy. The most serious problem is the excessive time the free vibration analysis spends being resident in the computer (wall clock time).

In the present paper the computations are carried out with different elgensolution routines on a number of large size nuclear plant structural models. A number of performances characteristics are recorded to offer some basis of comparison for the user of such algorithms. 
The analysis of nuclear plant building structures to be discussed here alms for the generation of displacement and acceleration response spectra which are to be used in the analysis of internal secondary structures. The most popular approach proceeds through a modal analysis requiring, as an initial step, the determination of the free vibration characteristics of the entire system. In recent years the systems to be analyzed have grown in complexity. The frequency range has been drastically expanded. This has been done in an effort to keep the numerical model close to the realities of the structural configuration and to the loading time histories.

In a modal time history analysis of such a system the main burden of the numerical computation lies with the determination of the free vibration characteristics. Cost and accuracy both play a major role. A number of improved elgensolution routines have been offered on the software market to address this situation.

The present paper explores cost and accuracy of a number of elgensolution routines which have been applied to models of nuclear plant bullding structures with several thousiand degrees of freedom and with several hundred free vibration modes and frequencies to be computed.

\section{EIGENSOLUTION METHODS}

Four principal elgensolution methods have been applied: determinant search methods; subspace 1teration methods without shift, w1th user-activated shift, and with progressive forward shifting; Lanczos QR-method; Householder QR-method. Cost saving by exploiting model symmetry is considered.

\subsection{DETERMINANT SEARCH}

The computational procedure of the determinant search method [1] consists of five bullding blocks; secant 1teration and Sturm sequence check; inverse 1teration; Gram-Schmid orthogonalization of the last six calculated elgenvectorg; Raylelgh quotient shift if convergence is slow; and computation of error bounds. The procedure has been avallable [ 2 ] In the subroutine SECANTD in which the decomposition $\underline{K}=\underline{L} \underline{D} \underline{L}^{T}$ is carried out by the subroutine BANDET without the use of secondary storage. While this solution algorithm is relatively fast it is limited by the avallable primary storage capacity. For large systems consisting of several thousand degrees of freedom and a half-bandwidth of several hundred, a secondary storage scheme is mandatory. An efficlent scheme 1s the skyline, column-wise storage of a sparse matrix in blocks. The corresponding solution algorithm is called COLDET. The details of skyline decomposition are explained in [3].

\subsection{SUBSPACE ITERATION}

The subspace 1teration procedure has been available for some time [2]. Its application to large elgenvalue problems, that 1s, a large number 
of requested eigenpairs $\left(\omega_{j}, \Phi_{j}\right)$ of structural models with a large number of equations and a large half-bandwidth exhibits a number of serious drawbacks. If there are $\mathrm{m}$ eigenvalues to be computed, conventional subspace iteration transforms to a subspace stiffness and mass matrix (see Tables 1 and 2)

$$
\underline{K}^{*}=\underline{\tilde{x}}^{\mathrm{T}} \underline{\mathrm{K}} \underline{\tilde{\mathrm{X}}}, \quad \underline{\mathrm{M}}^{*}=\underline{\tilde{\mathrm{X}}}^{\mathrm{T}} \underline{\mathrm{M}} \underline{\tilde{\mathrm{X}}}
$$

where $\underline{K}^{*}$ and $\underline{M}^{*}$ are symmetric but fully populated. $\underline{K}$ is the system stiffness matrix, $\underline{M}$ is the diagonal system mass matrix and $\underline{\tilde{X}}$ contains the approximation vectors of $m$ trial vectors. The storage requirement for the solution of the transformed eigenvalue problem with $\underline{K}^{*}$ and $\underline{M}^{*}$ by the Jacobi method is $2 \mathrm{~m}(\mathrm{~m}+1)$. When the total available primary storage is limited, the maximum size of the subspace is fixed. With $\mathrm{m}$ being very large in addition to a number of equations $n$ and a large half-bandwidth $m_{k}$ of the system stiffness matrix K another storage problem arises with the solution of the linear equations

$$
\underline{\mathrm{K}} \tilde{\mathrm{X}}=\underline{\mathrm{Y}} \text {. }
$$

The stiffness matrix $\underline{K}$ and the vectors $\widetilde{X}$ and $\underline{Y}$ are blocked so that $n_{b} \cdot n_{e q b}$ $\mathrm{n}$, where $\mathrm{n}_{\mathrm{b}}$ is the number of blocks and $\mathrm{n}_{\mathrm{eqb}}$ is the number of equations in a block. To cut down on the input/output operations during the solution of eq. (2) It is important to make $\mathrm{n}$ eqb as large as possible. If the m vectors are not all processed at once the block size can be increased thus expanding slightly the size limitation for the eigensolution procedure.

The most serious drawback of the subspace iteration, however, is the slow convergence when the size of the subspace 1s large [4]. A given range of frequencies to be explored in a model can be divided into a number of small subspaces when a shift is used. The shifted eigenvalue problem $\left(\omega_{j}^{2}=\mu\right.$ $\left.+\gamma_{j}\right) \underline{\hat{\mathrm{K}}} \underline{\Phi}=\underline{\gamma} \underline{\underline{M}} \underline{\underline{\Phi}}$

with $\hat{K}=\underline{K}-\mu \underline{M}$ (where $\mu$ is the shift value) is then solved for a given irequency range, with a known number of frequencies to be determined. The size of the subspace, $\mathrm{m}$, can then be kept at a level where no storage and accuracy problems arise.

User Activated Shift. - A solution procedure in which the user activates the shift ( $\mathrm{Table} 1$ ) allows the large eigensolution problem to be solved at a leisurely pace determined by the avallable primary storage. By permitting overlap of the frequency ranges analyzed the problems of bad convergence at the upper end of each subspace can be controlled. Also, the shift value can be chosen at a convenient point within the frequency range in order to accelerate the convergence. The results of the individual subspace iteration solutions are merged in the end on one file for use in the subsequent modal solution algorithms.

Progressive Forward Shifting. - An automatic shifting procedure has been developed in Ref.[5]. The solution procedure is shown in Table 2. The subspace is kept at $12 \leqslant m \leqslant n(1 / 2)$. The shifting strategy determines when it 
is appropriate to perform a shift based on the number of operations required for an 1teration without or with a new shift. The program determines what new shift value should be used. The number of operations is based on the convergence behavior of the current and previous iteration. When $i_{t} 1_{t e r a-}$ tions are needed before the next eigenvalue converges then a new shift is performed if $n_{o p d}+2 n_{o p c}{ }^{<1} t^{n}{ }_{o p c}$, where $n_{o p d}$ is the number of operations for one matrix decomposition and $n_{\text {opc }}$ is the number of operations for one 1teration cycle. The new shift value $\mu$ is chosen just below a projection of the next unconyerged eigenvalue for 1teration $k+1$,

$$
\lambda_{\text {proj }}=\frac{\lambda_{\mathrm{k}-1}-\lambda_{\mathrm{k}-2} \lambda_{\mathrm{k}}}{2 \lambda_{\mathrm{k}-1}-\lambda_{\mathrm{k}-2}-\lambda_{\mathrm{k}}} \text {. }
$$

Here lies one of the drawbacks of this shift strategy. When there are large clusters of closely spaced frequencies, as found, for example, in bullding models no two frequencies can be found far enough apart for a shift to be performed. If a shift is forced within the convergence radius of a previously converged eigenvalue, the logic of this procedure cannot cope with elgenvalues computed twice. This is the situation described in Ref $[4]$. It may be overcome by choosing $\mu$ between two elgenvalues which have converged to a high tolerance instead of between a converged and an unconverged one.

\subsection{LANCZOS QR}

The Lanczos QR-method is described in a number of References $[6,7,8]$. The solution procedure is summarized in Table 3 . The original eigenvalue problem is tranformed to

$$
(\underline{A}-\underline{\lambda} \underline{I}) \underline{Z}=0
$$

in which A Is a symmetric, tridiagonal matrix of order $m$ and I 1 s the unit matrix. Approximately the lower half of the $m$ eigenvalues computed are useable while the other eigenvalues are unacceptable. Since the formation and the solution of eq. (5) cannot be carried out in primary storage careful planning of secondary storage operations for transforming the original e1genvalue problem, solving the tridiagonal elgenproblem, and transforming the solution vectors $\underline{Z}$ back to the eigenvectors $\Phi$ is 1mportant. The complete set of $\underline{z}$-vectors would require $\mathrm{m}^{2}$ storage spaces. If $\mathrm{m}$ is twice the number of required frequencies, this number becomes prohibitively large, thus secondary storage is mandatory. For the computation of each of the $m$ Lanczos vectors $\underline{V}$ the complete blocked, decomposed stiffness matrix $\underline{K}=\underline{L} \underline{\mathrm{D}} \underline{L}^{\mathrm{T}}$ has to be passed through the subroutine REDVK during forward reduction and back substitution. This accounts for the major part of the computational effort. While this takes place the computation of the tridiagonal matrix coefficlents is being completed. Orthogonalizing and storing of the Lanczos vectors accounts for the moving of approximately $m^{2} n$ words. An additional $m \cdot n$. $\mathrm{n}_{\mathrm{b}}$ words are moved during the retransformation from the $\underline{Z}^{\prime} s$ to the $\underline{\phi}^{\prime} s$

$$
\underline{\phi}=\underline{\mathrm{V}} \underline{\mathrm{z}}
$$


where $\Phi$ and $\underline{V}$ are $n$ by $m$ and $\underline{Z}$ is an $m$ by m matrix. As will be seen in the case studies the formation of the tridiagonal matrix accounts for the 1 argest part of the computational effort and of the input/output activity on secondary storage.

\subsection{HOUSEHOLDER QR}

The Householder QR-method is similar to the Lanczos method in that it transforms the original eigenvalue problem with the system matrices $\underline{K}$ and $\underline{M}$ to the tridiagonalized problem of eq. (5). The difference is in the size of the tridiagonalized problem: Lanczos operates with $m$ vectors, where in is aporoximately twice the number of requested eigenpairs while Householder uses all $n$ vectors (see Ref.[1] pp.460-473). The storage required is of the order $n(n+1) / 2$. For large models this primary storage requirement cannot be met. Therefore the Householder QR-method in the present form cannot be used for large building type models.

\subsection{EXPLOITING MODEL SYMMETRY}

There are a number of cases in which the model exhibits at least one symmetry plane (Fig.1). The symmetry is exploited for drastic cost reduction by using a half-model with $\underline{K}_{H}$ and $\underline{M}_{H}$. Two elgenproblems have to be solved

$$
\left(\underline{K}_{S}-\underline{\Omega}_{S}^{2} \underline{M}_{S}\right) \underline{\Phi}_{S}=0 \quad \text { and } \quad\left(\underline{K}_{A}-\underline{\Omega}_{A} \underline{M}_{A}\right) \underline{\Phi}_{A}=0
$$

The Indices $S$ and $A$ corresoond to the symmetry and antisymmetry conditions, respectively, on the symmetry plane. For the model on F1g.1 they are (see Ref.[g])

$$
\mathrm{u}_{\mathrm{x}}=\mathrm{w}_{\mathrm{y}}=\mathrm{w}_{\mathrm{z}}=0 \quad \text { (S) and } \mathrm{u}_{\mathrm{y}}=\mathrm{u}_{\mathrm{z}}=\mathrm{w}_{\mathrm{x}}=0 \quad \text { (A) . }
$$

The solution of the two eigenproblems of eq. $(7,8)$ delivers the complete modes of the half model $(\mathrm{H})$ and the reflected model (R)

$$
\Phi=\left[\begin{array}{c}
\underline{\Phi}_{\mathrm{H}} \\
\hdashline \Phi_{\mathrm{R}}
\end{array}\right]=\left[\begin{array}{c:c}
\underline{\Phi}_{\mathrm{S}} & \underline{\Phi}_{\mathrm{A}} \\
\hdashline \underline{\Phi}_{\mathrm{S}} & -\underline{\Phi}_{\mathrm{A}}
\end{array}\right] \text {. }
$$

When the generalized masses are computed,

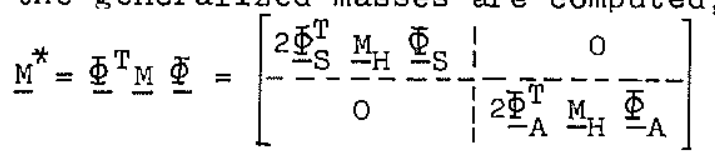

It becomes abvious that no other modes exist.

A time history analysis with loads on the halfmodel, $\underline{P}_{H}$, and on the reflected model, $\underline{P}_{R}$, is carried out by considering the symmetric and the antisymmetric half-model separately. The half-model load components are

$$
\underline{P}_{S}=\left(\underline{P}_{H}+\underline{P}_{R}\right) / 2, \quad \underline{P}_{A}=\left(\underline{P}_{H}-\underline{P}_{R}\right) / 2,
$$

and the results (displacements, accelerations, stresses) are

$$
\underline{\mathrm{V}}_{\mathrm{S}}=\underline{\Phi}_{\mathrm{S}} \underline{\mathrm{Y}}_{\mathrm{S}}(\mathrm{t}), \quad \underline{\mathrm{V}}_{\mathrm{A}}=\underline{\Phi}_{\mathrm{A}} \underline{\underline{Y}}_{\mathrm{A}}(\mathrm{t}) \text {. }
$$


The final results for the half-model and the reflected model are obtalned by superposition:

$$
\underline{\mathrm{V}}_{\mathrm{H}}=\underline{\mathrm{V}}_{\mathrm{S}}+\underline{\mathrm{V}}_{\mathrm{A}}, \quad \underline{\mathrm{V}}_{\mathrm{R}}=\underline{\mathrm{V}}_{\mathrm{S}}-\underline{\mathrm{V}}_{\mathrm{A}} \text {. }
$$

The reflected model is found from the half-model by mirror-imaging the nodes and elements on the symmetry plane.

The savings of solving the two half-models of eq.(12) are dramatic in light of $n, m_{k}$ and $m$ all belng approximately half of the full model.

\section{BUILDING MODELS}

\subsection{MODEL CHARACTERISTICS}

The bullding models (see Table 4) which have been analyzed consist of truss, beam, plate, and uncoupled and coupled spring elements. Model 1 (Table 4) is an internal structure with additional pipe elements. It has been used for checking solution times of the varlous solution methods mentioned in this paper. The other models are typical bullding models with a large number of equations and a large half-bandw1dth. In the bullding models only the translational degrees of freedom carry any mass. Approximately five percent of the total degrees of freedom do not carry any stiffness. This 1 d de to the lack of rotational stiffness normal to the plate surface. of the 2754 total degrees of freedom of model 5, there are 1377 mass dof, and 2636 stiffness dof. The excess dof are automatically restrained.

\subsection{COMPARISON OF EIGENSOLUTIONS}

In Table 4 only two solution methods are compared, the Lanczos QRmethod (LQR) and the subspace iteration with progressive forward shifting (SPFS). From the comparison of models 5 through 8 it is apparent that LQR is supertor to SPFS even when only 10 frequencles are being computed. Th1s is true for a small field length (work area 29500 words) and a large field length (work area 55000 words). The LQR total solution time is only between 50 and 60 percent of the SPFS time. This is the reason for favoring the application of the LQR solution method. All solution results have been checked with STARDYNE results for accuracy by comparing frequencles and mode shapes, generalized welghts, composite damping, modal participation factors and modal welghts.

\subsection{COMPUTATIONAL PERFORMANCE}

The performance characterlstics can be examined from the CPA, I/O, CM, SS and wall clock time readings. Models 2 and 3 differ in total dof by 14 percent (based on the smaller model) and have the same half-bandw1dth. For the larger model 50 percent more modes and frequencles have been requested. All performance parameters have more than doubled. An 1mportant performance flgure is the ratio of wall clock time over total solution time. Th1s ratio is approximately 10 and more for the LQR method. 
Of all the elgensolution algorlthms considered in this paper the Lanczos QR-method is the most promising one. By further reduction of the amount of data transfer between primary and secondary storage, particularly during tridiagonalization, the method can be extended for extrenely large models. The most important area of urgently needed improvements is the reduction of the time the computer run is resident in the computer (wall clock time).

\section{REFERENCES}

[1] BATHE, K.J. and WILSON, E.L., Numerical Methods In Finite Element Analysis, Prentice Hall, Inc., Englewood Cliffs, N.J., 1976.

[2] BATHE, K.J., WILSON, E.L., and PETERSON, F.E., "SAP4 - A Structural Analysis Program for Static and Dynamic Response of Linear Systems", Report EERC 73-11, College of Engineering, University of California, Berkeley, June 1973, revised Apr. 1974.

[3] BATHE, K.J., "ADINA - A Finite Element Program for Automatic Dynamic Incremental Nonlineax Analysis", Report 82448-1, Acoustics and Vibration Laboratory, Department of Mechanical Engineering, Massachusetts Institute of Technology, Cambridge, Mass., 1975, revised Dec. 1978.

[4] BATHE, K.J., and RAMASWANY, S., "An Accelerated Subspace Iteration Method", Computer Methods in Applied liechanics and Engineering, Vol. 23 , pp. 313-331, 1980.

[5] ITOH, T., "Subsoace Iteration w1th Progressive Forward Shifting and its Simulation Analysis", SESM CE29ᄋ Report 689, C1v11 Engineering Department, University of California, Berkeley, 1978.

[6] CHOWDHURY, P.C., "The Truncated Lanczos Algorithm for Partial solution of the Symmetric Eigenproblem", Computers and Structures, Vol.6, pp.439-446, 1 : 76 .

[7] NEWMAN, M., and FLANAGAN, P.F., "Eigenvalue Extraction In NASTRAN by the Tridiagonal Reduction (FEER) Method - Real Eigenvalue Analysis", NASA CR-2731, Analytical Mechanics Associates, Jericho, New York, August 1976.

[8] NEWMAN, M., and PIPANO, A., "Fast Modal Extraction in NASTRAN via the FEER Computer Program", in NASTRAN: User's Experience, Paper No. N7414608 , Langley Research Center, Hampton, Virginia, Report No. NASA TM X-2893, September, 1973.

[9] LEIMBACH, K.R., and FRANZ, D., Discussion of "Symmetry in Structura] Mechanfos" by P.G. Glockner, Journal of the Structural Division, Vol. 9., No.ST8, August, 1973 , pp.1792-1794. 
Table 1 - Subspace Iteration with User-In1tiated Sh1ft

\begin{tabular}{|c|c|c|}
\hline Step & Operation & Computation \\
\hline 1 & $\begin{array}{l}\text { Establish frequengy range } \\
\mu_{\min }=\omega_{\min }^{2} \mu_{\max }=\omega_{\max }^{2}\end{array}$ & $\begin{array}{l}\hat{K}_{\max }=\frac{K}{K}-\mu_{\max } \frac{M}{M} \\
\underline{\underline{K}}_{\min }=\underline{M}-\mu_{\min }\end{array}$ \\
\hline 2 & $\begin{array}{l}\text { Count number of frequencles } \\
\text { in range by } \underline{\hat{K}}=\underline{L} \underline{D} \underline{L}\end{array}$ & $\Delta \mathrm{m}=\mathrm{m}_{\max }{ }^{-m_{\mathrm{m} 1 \mathrm{n}}}$ \\
\hline 3 & Perform $\operatorname{sh} 1 f t \mu \approx \frac{1}{2}\left(\mu_{\min }+\mu_{\max }\right)$ & $\underline{\hat{K}}=\underline{K}-\mu \frac{M}{m}$ \\
\hline 4 & Factorize $\widehat{\mathrm{K}}$ & $\underline{\underline{K}}=\underline{L} \underline{\underline{D}} \underline{L}^{\mathrm{I}}$ \\
\hline \multicolumn{3}{|c|}{ for 1ter } \\
\hline 5 & $\begin{array}{l}\text { Set trial vectors } \underline{X}_{k} \\
\text { Compute Inertia forces } \underline{Y}_{k}\end{array}$ & $\underline{\mathrm{Y}}_{\mathrm{k}}=\underline{\mathrm{M}} \underline{\mathrm{x}}_{\mathrm{k}}$ \\
\hline 6 & $\begin{array}{l}\text { Solve for improved trial } \\
\text { vectors in subblocks } a, \ldots, g\end{array}$ & {$\left[\underline{x}_{g}\right]_{k}$} \\
\hline$\sqrt{7}$ & Form subspace stiffness & $\overrightarrow{\mathrm{K}}^{*}=\tilde{\mathrm{X}}_{\mathrm{k}}^{\mathrm{T}} \overline{\mathrm{Y}}_{\mathrm{N}}$ \\
\hline 8 & Improved inertia forces & $\underline{\widetilde{Y}}_{\mathrm{k}}=\frac{\mathrm{M}}{\sim} \frac{\widetilde{\mathrm{X}}}{\sim} \mathrm{k}$ \\
\hline 9 & Form subspace mass & $\underline{M}^{*}=\tilde{X}_{k}^{T} \underline{\tilde{Y}}_{k}$ \\
\hline 10 & $\begin{array}{l}\text { Solve subspace elgenvalue problem } \\
\text { w1th Jacob1 Method }\end{array}$ & {$\left[\left(\underline{K}^{*}+\underline{M}^{*}\right)-\underline{\Omega}^{2} \underline{M}^{*}\right) \underline{q}^{*}=0$} \\
\hline 11 & Compute improved vectors & $\underline{x}_{k}=\tilde{x}_{k} \underline{q}^{*}$ \\
\hline 12 & $\begin{array}{l}\text { Check tolerance. for all elgen- } \\
\text { values } \omega_{j} \quad j=1, \ldots, m\end{array}$ & $\varepsilon_{j}=\frac{\omega_{j, k-1}^{2}-\omega_{j, k}^{2}}{\omega_{j, k}^{2}} \leq$ tol \\
\hline 13 & If tolerance is not met got to 5 & $\bar{x}_{\mathrm{k}} \rightarrow \underline{x}_{\mathrm{k}+1}$ \\
\hline 14 & Complete solution & $\omega_{j, k} \rightarrow \omega_{j} \quad \underline{x}_{k} \longrightarrow \underline{\Phi}$ \\
\hline
\end{tabular}

Table 2 - Subspace Iteration with Progressive Forward Shifting

\begin{tabular}{|c|c|c|}
\hline Step & Operation & Computation \\
\hline $\begin{array}{l}1 \\
2\end{array}$ & $\begin{array}{l}\text { Perform shift } \\
\text { Factorlze } \underline{\hat{K}}\end{array}$ & $\begin{array}{l}\frac{\hat{K}}{\hat{K}}=\underline{K}-\mu \frac{M}{T} \\
\underline{L}=\underline{D} \underline{L}^{-}\end{array}$ \\
\hline \multicolumn{3}{|c|}{ for 1 teration $\mathrm{k}$ : } \\
\hline 3 & $\begin{array}{l}\text { Using trlal vectors } \underline{X}_{k} \\
\text { compute inertia forces } \underline{Y}_{k}\end{array}$ & $\begin{array}{l}\underline{Y}_{\mathrm{k}}=\underline{\mathrm{M}} \underline{\mathrm{x}}_{\mathrm{k}} \\
\hat{\mathrm{K}} \tilde{\mathrm{X}}=\mathrm{Y}\end{array}$ \\
\hline $5^{4}-$ & Solve for 1mproved trial vectors & $\underline{\underline{K}} \underline{X}_{\mathrm{k}}=\underline{Y}_{\mathrm{k}}$ \\
\hline 5 & $\begin{array}{l}\text { see steps } 7 \text { through } 12 \text { In Table } 1 \\
\text { Form and solve transformed and shifted } \\
\text { compute 1mproved vectors_and check tole }\end{array}$ & $\begin{array}{l}\text { Igenvalue problem, } \\
\text { ances }\end{array}$ \\
\hline 11 & $\begin{array}{l}\text { Replace converged eigenvectors } \\
\text { with random vectors, orthogonalize } \\
\text { w1th respect to converged vectors } \\
\text { to form new trial subspace, } x_{k+1}\end{array}$ & $\underline{x}_{k} \rightarrow \underline{x}_{k+1}$ \\
\hline 12 & $\begin{array}{l}\text { Compete new shift } \\
\text { shift strategy determines whether } \\
\text { to go to } 1 \text { or to go to } 3 \text {. }\end{array}$ & $\mu_{\text {new }}$ \\
\hline 13 & Complete solution & $\omega_{j, k} \rightarrow \omega_{j} \quad \underline{x}_{k} \rightarrow \underline{\Phi}$ \\
\hline
\end{tabular}


Table 3 - Lanczos QR 1)

\begin{tabular}{|c|c|c|}
\hline Step & Operation & Computation \\
\hline 1 & Factorize $\underline{K}$ & $\underline{K}=\underline{L} \underline{D} \underline{L}^{T}$ \\
\hline \multicolumn{3}{|c|}{ for each vector $j \quad j=1, \ldots, m$} \\
\hline 2 & $\begin{array}{l}\text { Generate random vector } \underline{X}, j \\
\text { and compute inertia force } \underline{Y} \text {. }\end{array}$ & $\underline{Y}_{j}=\underline{M} \underline{X}_{j}$ \\
\hline 3 & Compute Lanczos vector $\widetilde{V}_{\mathrm{J}}$ & $\underline{K}_{\underline{1}} \underline{\tilde{V}}_{i}$ \\
\hline 4 & Normalize Lanczos Vector & $\underline{v}_{j}=\frac{\underline{v}_{j}}{\left(\underline{\tilde{V}}_{j}^{\mathrm{T}} \underline{\mathrm{M}} \underline{\tilde{V}}_{j}\right)^{1 / 2}}$ \\
\hline 5 & $\begin{array}{l}\text { Check orthogonality of trial vector with } \\
\text { all previously computed vectors } 1=1, \ldots \\
1=1, \ldots, j-1\end{array}$ & $M_{i j}^{*}=\underline{V}_{i}^{T} \underline{M} \underline{V}_{j}$ \\
\hline 6 & $\begin{array}{l}\text { If not orthogonal, reorthogonalize } \\
\text { iteratively (k } \leqslant 14 \text { iterations) }\end{array}$ & $\underline{\hat{v}}_{j}^{\mathrm{k}}=\tilde{\tilde{V}}_{j}^{\mathrm{k}-1}-\sum_{i=1}^{\mathrm{J}} \sum_{i j}^{-\perp} M_{i}^{*} \underline{v}_{i}$ \\
\hline 7 & Normalize orthogonal Lanczos Vector & $\underline{v}_{j}^{\mathrm{k}}=\frac{-\mathrm{J}}{\left[\left(\mathrm{v}_{j}^{\mathrm{k}}\right)^{\mathrm{T}} \mathrm{M}_{j}^{\mathrm{k}}\right]^{\overline{1 / 2}}}$ \\
\hline 8 & Compute second Lanczos Vector & $\underline{K}_{\underline{W}}=\underline{\mathrm{V}}_{j}^{\mathrm{k}}$ \\
\hline 9 & Compute off-diagonal coefficient & $\beta_{j}=\underline{w}_{j}^{T} \underline{M}_{j-1}$ \\
\hline 10 & Compute diagonal coefficient & $\alpha_{j}=w_{j}^{T} \underline{M}_{j}$ \\
\hline 11 & Compute new Lanczos Vector & $\underline{\tilde{V}}_{j+1} \quad \underline{V}_{j}-\alpha_{j} \frac{V}{\tilde{V}}_{j}-\beta_{j} \underline{V}_{j-1}$ \\
\hline 12 & Normalize new Lanczos Vector & $\underline{v}_{j+1}=\frac{j+1}{\left(\underline{v}_{j+1}^{T}-\underline{\tilde{v}}_{j+1}\right)^{j / 2}}$ \\
\hline 13 & Solve tridiagonalized $m$ by $m$ eigenproblem & $(\underline{A}-\underline{\lambda} \underline{I}) \underline{Z}=\underline{Q}$ \\
\hline 14 & $\begin{array}{l}\text { Transform back to original } m \text { by } n \\
\text { vector space from } \\
\text { m by m tridiagonalized problem space } \\
\text { using Lanczos vectors }\end{array}$ & $\begin{array}{l}\omega_{f}^{2}=\frac{1}{\lambda_{f}} \\
\underline{\Phi}=\underline{\mathrm{V}} \underline{\mathrm{Z}}\end{array}$ \\
\hline 15 & Check tolerances (eq.27a,Ref.[7]) & $\varepsilon_{j} \leqslant$ tol \\
\hline
\end{tabular}

1) $\operatorname{Ref} .[7], \mathrm{pp}, 35-43$

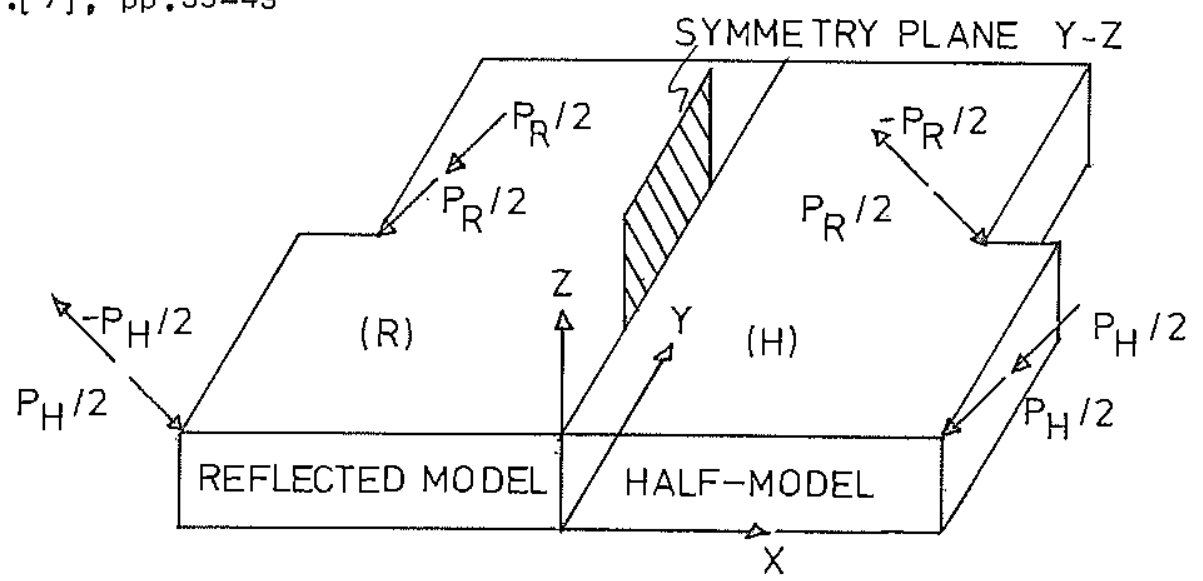

Fig.1 - Model with one Symmetry Plane 


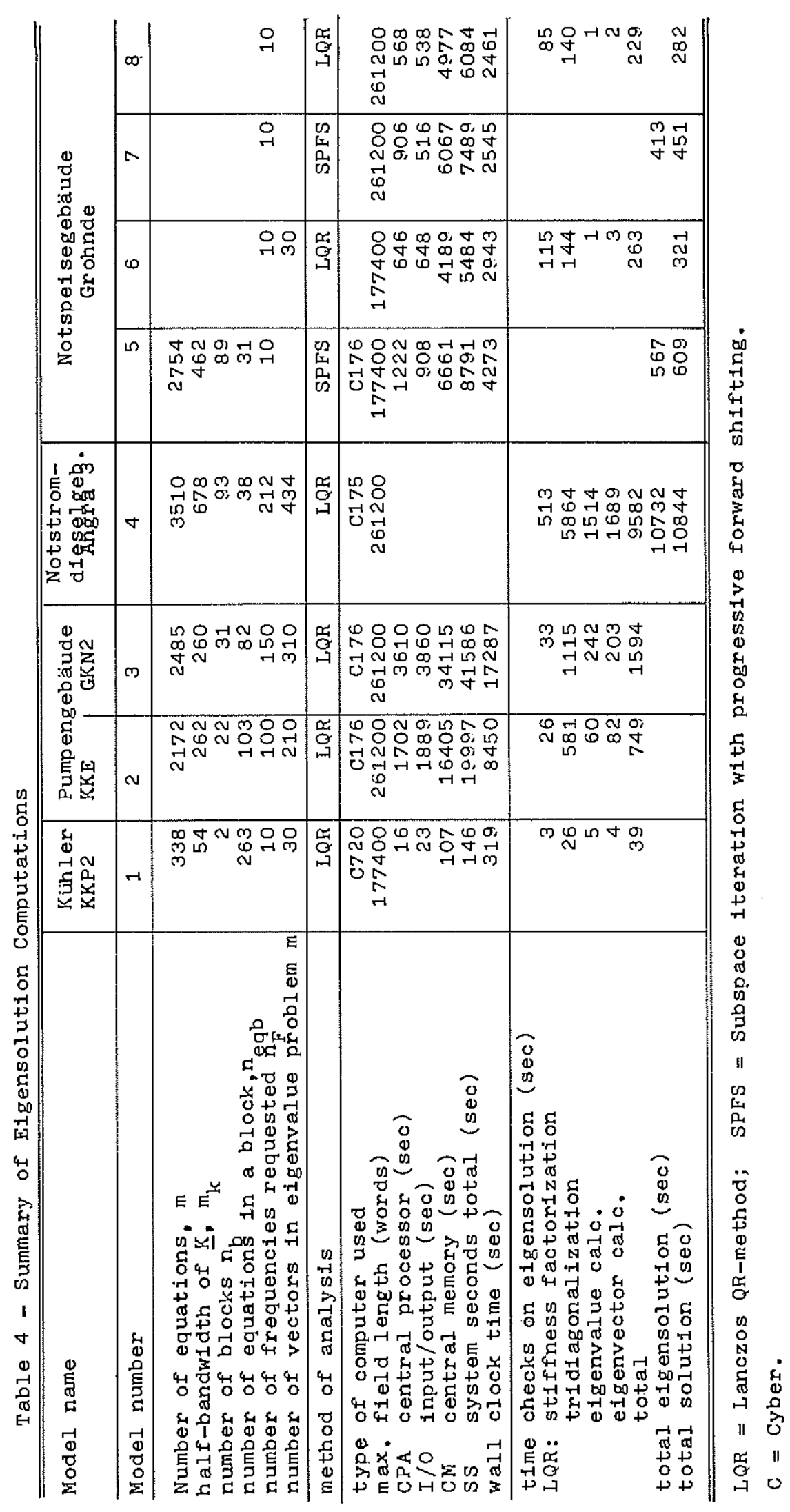

